\title{
Optimal Numerical Differentiation Using Three Function Evaluations
}

\author{
By J. Marshall Ash and Roger L. Jones
}

Abstract. Approximation of $f^{\prime}(x)$ by a difference quotient of the form

$$
h^{-1}\left[a_{1} f\left(x+b_{1} h\right)+a_{2} f\left(x+b_{2} h\right)+a_{3} f\left(x+b_{3} h\right)\right]
$$

is found to be optimized for a wide class of real-valued functions by the surprisingly asymmetric choice of $\mathrm{b}=\left(b_{1}, b_{2}, b_{3}\right)=(1 / \sqrt{3}-1,1 / \sqrt{3}, 1 / \sqrt{3}+1)$. The nearly optimal choice of $b=(-2,3,6)$ is also discussed.

1. Introduction. The problem of best approximating the derivative of a function at a single point using two values of the function is "best" solved by using the difference quotient

$$
d_{0}(h)=\frac{f\left(x+\frac{1}{2} h\right)-f\left(x-\frac{1}{2} h\right)}{h} .
$$

We consider the same problem using three values of the function and arrive at three different solutions by interpreting "best" in five different ways. Our best difference quotients are

$$
\begin{gathered}
d_{1}(h)=\frac{(3-2 \sqrt{3}) f\left(x+\left(\frac{1}{\sqrt{3}}+1\right) h\right)+4 \sqrt{3} f\left(x+\frac{1}{\sqrt{3}} h\right)-(3+2 \sqrt{3}) f\left(x+\left(\frac{1}{\sqrt{3}}-1\right) h\right)}{6 h}, \\
d_{2}(h)=\frac{f(x+h)+\omega^{2} f(x+\omega h)+\omega f\left(x+\omega^{2} h\right)}{3 h},
\end{gathered}
$$

where $\omega=-\frac{1}{2}+\sqrt{3} i / 2$ and $\omega^{2}=-\frac{1}{2}-\sqrt{3} i / 2$ are the cube roots of 1 , and

$$
d_{3}(h)=\frac{32 f(x+3 h)-27 f(x-2 h)-5 f(x+6 h)}{120 h} .
$$

Section 3 below was motivated by the kind suggestion of J. Lyness.

2. Minimum Truncation Error. Whenever we write $f$, we will mean either a complex-valued function of a complex variable or a real-valued function of a real variable. In the former case we assume that $f$ is analytic near $x$ and in the latter case that $f$ is five times differentiable at $x$. The point $x$ will be fixed and the variable $h$ will be small. Our assumptions are chosen to guarantee (i) that $f$ have at $x$ a Taylor expansion to order $h^{4}$ with error $O\left(|h|^{5}\right)$ and (ii) that there be a bound for the modulus of $f^{\prime \prime \prime \prime}$ near $x$.

Received May 2, 1980; revised October 10, 1980.

1980 Mathematics Subject Classification. Primary 65D25; Secondary 65G05, 39A05.

Key words and phrases. Numerical differentiation, discretization error, truncation error, generalized difference quotient. 
Consider the general three-point difference quotient

$$
d(h)=h^{-1} \sum_{i=1}^{3} a_{i} f\left(x+b_{i} h\right)=d(\mathbf{a}, \mathbf{b})(h) .
$$

Substituting Taylor's theorem,

$$
f\left(x+b_{i} h\right)=\sum_{j=0}^{4} \frac{f^{(j)}(x)}{j !} h^{j} b_{i}^{j}+\frac{f^{(5)}\left(\xi_{i}\right)}{5 !} h^{5} b_{i}^{5},
$$

into (5) and interchanging the order of summation gives

$$
\left\{\begin{array}{l}
d(h)=\sum_{j=0}^{4} c_{j} \frac{f^{(j)}(x)}{j !} h^{j-1}+O\left(h^{4}\right), \\
c_{j}=\sum_{i=1}^{3} a_{i} b_{i}^{j}, \quad j=0,1,2,3,4 .
\end{array}\right.
$$

For $d(h) \rightarrow f^{\prime}(x)$ as $h \rightarrow 0$, we must have $c_{0}=0$ and $c_{1}=1$. This motivates our first definition.

Definition 1. A three-point rule for the first derivative is a difference quotient $d(\mathbf{a}, \mathbf{b})(h)$ of the form (5) where $c_{0}=\sum a_{i}=0$ and $c_{1}=\sum a_{i} b_{i}=1$.

Definition 2. A three-point rule for the first derivative is of degree $k$ if and only if $d(\mathbf{a}, \mathbf{b})(h)=f^{\prime}(0)$ for every polynomial $f$ of degree $k$.

LEMMA 1. A necessary and sufficient condition for the three-point rule to be of degree 3 is

$$
\left\{\begin{array}{l}
c_{0}=\sum a_{i}=0 \\
c_{1}=\sum a_{i} b_{i}=1, \\
c_{2}=\sum a_{i} b_{i}^{2}=0 \\
c_{3}=\sum a_{i} b_{i}^{3}=0 .
\end{array}\right.
$$

The proof of this is immediate from (6) above.

LEMMA 2. No three-point rule is of degree 4. For a three-point rule to be degree 3, we must have all $b_{i}$ distinct.

Proof. For a rule to be of degree 4 would require $c_{2}=c_{3}=c_{4}=0$. Write this as a matrix equation

$$
B \mathbf{a}=\left(\begin{array}{lll}
b_{1}^{2} & b_{2}^{2} & b_{3}^{2} \\
b_{1}^{3} & b_{2}^{3} & b_{3}^{3} \\
b_{1}^{4} & b_{2}^{4} & b_{3}^{4}
\end{array}\right)\left(\begin{array}{l}
a_{1} \\
a_{2} \\
a_{3}
\end{array}\right)=\left(\begin{array}{l}
0 \\
0 \\
0
\end{array}\right)
$$

If all $b_{i} \neq 0$, then the Vandermonde-like matrix $B$ is nonsingular, so $\mathbf{a}=\mathbf{0}$, contrary to $c_{1}=1$. If, say, $b_{3}=0$, the three equations $c_{2}=c_{3}=0, c_{1}=1$ lead quickly to a similar contradiction.

Similarly, if, say, $b_{2}=b_{3}$, we may as well combine $a_{2}$ and $a_{3}$, which implies we are dealing with a two-point rule. As above, if both $b_{i} \neq 0$, the two equations $c_{2}=c_{3}=0$ force a to be zero, contrary to $c_{1}=1$. The case of $b_{2}=b_{3}=0$ is treated similarly. Thus, for a three-point rule to be of degree 3 , we must have all $b_{i}$ distinct. 
LEMMA 3. The three-point rule $d$ is of degree 3 if and only if

$$
b_{1} b_{2}+b_{1} b_{3}+b_{2} b_{3}=0 \text {. }
$$

Proof. The first three equations in (7), $c_{0}=c_{2}=0, c_{1}=1$, may be written as a matrix equation

$$
B \mathbf{a}=\left(\begin{array}{l}
0 \\
1 \\
0
\end{array}\right),
$$

where the Vandermonde matrix $B$ is easily inverted. Substitute the result of this,

$$
\mathbf{a}=\left[\left(b_{2}-b_{1}\right)\left(b_{3}-b_{1}\right)\left(b_{3}-b_{2}\right)\right]^{-1}\left(b_{2}^{2}-b_{3}^{2}, b_{3}^{2}-b_{1}^{2}, b_{1}^{2}-b_{2}^{2}\right),
$$

into $c_{3}=0$ to obtain (8).

Definition 3. A three-point rule $d(\mathbf{a}, \mathbf{b})$ is said to be normalized if

$$
\min \left\{\left|b_{1}-b_{2}\right|,\left|b_{1}-b_{3}\right|,\left|b_{2}-b_{3}\right|\right\}=1 \text {. }
$$

Motivation for Definition 3. The mapping $h \rightarrow c h$ for a nonzero constant $c$, which may also be thought of as mapping (a, b) $\rightarrow\left(c^{-1} \mathbf{a}, c \mathbf{b}\right)$ while leaving $h$ fixed, obviously does not change anything of substance. In other words, the set of three-point rules $d(\mathbf{a}, \mathbf{b})(h)$ partitions into equivalence classes with $d(\mathbf{a}, \mathbf{b}) \sim$ $d\left(\mathbf{a}^{\prime}, \mathbf{b}^{\prime}\right)$ if there is a constant $c$ such that $\mathbf{a}^{\prime}=c^{-1} \mathbf{a}$ and $\mathbf{b}^{\prime}=c \mathbf{b}$. The quantity $c_{4}(\mathbf{a}, \mathbf{b})$ is not a class invariant, for if $d(\mathbf{a}, \mathbf{b}) \sim d\left(\mathbf{a}^{\prime}, \mathbf{b}^{\prime}\right)$, where $\left(\mathbf{a}^{\prime}, \mathbf{b}^{\prime}\right)=\left(\varepsilon^{-1} \mathbf{a}, \boldsymbol{\varepsilon} \mathbf{b}\right)$ with $0<\varepsilon<1$, we have

$$
\left|c_{4}\left(\mathbf{a}^{\prime}, \mathbf{b}^{\prime}\right)\right|=\left|\sum\left(\varepsilon^{-1} a_{i}\right)\left(\varepsilon b_{i}\right)^{4}\right|=\varepsilon^{3}\left|c_{4}(\mathbf{a}, \mathbf{b})\right|<\left|c_{4}(\mathbf{a}, \mathbf{b})\right| .
$$

By picking $\varepsilon$ very small, we can find arbitrarily small $c_{4}$ without really changing anything. Some normalization is required to keep the vector $\mathbf{b}$ from collapsing to 0 .

In difference quotient (1) or in the standard difference quotient $(f(x+h)-f(x)) / h$, a normalization has been achieved by expressing the error in powers of the distance between the two arguments. Set

$$
\delta=\min \left\{\left|b_{1}-b_{2}\right|,\left|b_{1}-b_{3}\right|,\left|b_{2}-b_{3}\right|\right\}
$$

and rewrite the dominant error term in (6) as

$$
c_{4} \frac{f^{(4)}(x)}{4 !} h^{3}=\left(\frac{c_{4}}{\delta^{3}}\right) \frac{f^{(4)}(x)}{4 !}(\delta h)^{3}=G \frac{f^{(4)}(x)}{4 !}(\delta h)^{3} .
$$

A simple calculation as in (9) above shows that $G=G(\mathbf{a}, \mathbf{b})$ is constant on each equivalence class.

LEMMA 4. All three-point rules of degree 3 are of the form

$$
d(t)=\frac{t^{3}(t+2) f(x+(1+t) h)-(1+2 t) f(x+t(1+t) h)-(t+1)^{2}\left(t^{2}-1\right) f(x-t h)}{t(2 t+1)(t+2)\left(t^{2}-1\right) h},
$$

for some value of $t$, or are obtained from (11) by a transformation $(\mathbf{a}, \mathbf{b}) \rightarrow\left(c^{-1} \mathbf{a}, c \mathbf{b}\right)$.

Proof. Select representatives from each equivalence class by setting $b_{1}=1$. Let $t=b_{2}$. From (8), $b_{3}=-t /(t+1)$. Clear fractions by sending $(\mathbf{a}, \mathbf{b}) \rightarrow$ $((1 /(t+1)) \mathbf{a},(t+1) \mathbf{b})$. Our representative quotient becomes $(11)$. 
THEOREM 1. Let $d$ be a normalized three-point rule of degree 3. Fix $h$ and restrict a and $\mathrm{b}$ to be real. Then truncation error is minimized when $d=d_{1}$. (See Eq. (2).) This minimizing rule is unique up to the transformation $(\mathbf{a}, \mathbf{b}) \rightarrow(-\mathbf{a},-\mathbf{b})$.

Proof. Let $d^{\prime}\left(\mathbf{a}^{\prime}, \mathbf{b}^{\prime}\right)$ be any normalized three-point rule of degree 3. By Lemma 1 , we have $c_{2}\left(\mathbf{a}^{\prime}, \mathbf{b}^{\prime}\right)=c_{3}\left(\mathbf{a}^{\prime}, \mathbf{b}^{\prime}\right)=0$, so to minimize truncation error we must minimize $\left|c_{\mathbf{4}}\left(\mathbf{a}^{\prime}, \mathbf{b}^{\prime}\right)\right|$. By Lemma 4 , there is a real number $t$ and an equivalent three-point rule $d(t)=d(\mathbf{a}(t), \mathbf{b}(t))$ of the form (11). Since

$$
\left|c_{4}\left(\mathbf{a}^{\prime}, \mathbf{b}^{\prime}\right)\right|=\left|c_{4}\left(\mathbf{a}^{\prime}, \mathbf{b}^{\prime}\right) / 1^{3}\right|=\left|G\left(\mathbf{a}^{\prime}, \mathbf{b}^{\prime}\right)\right|=|G(\mathbf{a}, \mathbf{b})|=\left|c_{4}(\mathbf{a}, \mathbf{b})\right| / \delta^{3},
$$

where $\delta=\min _{i \neq j}\left\{\left|b_{i}-b_{j}\right|\right\}$ (see (10)), we see that we must minimize $|G(t)|=$ $\left|c_{4}(\mathbf{a}(t), \mathbf{b}(t))\right| / \delta(\mathbf{b}(t))^{3}$ as $t$ varies over $\mathbf{R}$.

Substituting the values of $\mathbf{a}(t)$ and $\mathbf{b}(t)$, as given in (11), into $\left|c_{4}(\mathbf{a}, \mathbf{b})\right|$ reduces our problem to that of minimizing

$$
|G(t)|=\frac{\left|t^{2}(t+1)^{2}\right|}{\min \left\{\left|1-t^{2}\right|,|2 t+1|,|t(t+2)|\right\}^{3}}=\frac{\left|t^{2}(t+1)^{2}\right|}{\delta(t)^{3}}
$$

Since $t$ is real, we have

$$
\delta(t)=\left\{\begin{array}{l}
\left|t^{2}-1\right|,-(\sqrt{3}+1) / 2<t<-\sqrt{3}+1,(\sqrt{3}-1) / 2<t<\sqrt{3}+1, \\
\left|t^{2}+2 t\right|,-(\sqrt{3}+2)<t<-(\sqrt{3}+1) / 2, \sqrt{3}-2<t<(\sqrt{3}-1) / 2, \\
|2 t+1|,-\infty<t<-(\sqrt{3}+2),-\sqrt{3}+1<t<\sqrt{3}-2, \sqrt{3}+1<t<\infty .
\end{array}\right.
$$

Substituting this into (12) and using the methods of elementary differential calculus on each interval yields that

$$
\min _{t \in \mathbf{R}}|G(t)|=\frac{2}{9} \sqrt{3}
$$

and that this minimum occurs at $t=1+\sqrt{3}, 1-\sqrt{3}, \frac{1}{2}(\sqrt{3}-1), \frac{1}{2}(-\sqrt{3}-1)$, $\sqrt{3}-2$, and $-\sqrt{3}-2$. All six values of $t$ give equivalent difference quotients (the corresponding b vectors are scalar multiples of one another). Normalizing the difference quotient corresponding to $t=\frac{1}{2}(\sqrt{3}-1)$ by sending $(\mathbf{a}, \mathbf{b}) \rightarrow$ $((\sqrt{3} / 2) \mathbf{a},(2 / \sqrt{3}) \mathrm{b})$ produces difference quotient $d_{1}$ (see (2)) as the unique (up to $(\mathbf{a}, \mathbf{b}) \rightarrow(-\mathbf{a},-\mathbf{b}))$ normalized real three-point rule minimizing truncation error.

THEOREM 2. Let $d$ be a normalized three-point rule of degree 3. Fix $h$ but allow all variables and functions to be complex-valued. Then truncation error is minimized when $d=d_{2}$. (See Eq. (3).) This minimizing rule is unique up to the transformations $(\mathbf{a}, \mathbf{b}) \rightarrow\left(e^{-i \varphi} \mathbf{a}, e^{i \varphi} \mathbf{b}\right), 0<\varphi<2 \pi$.

Proof. As in the proof of Theorem 1, we must minimize the function $|G(t)|$ given in (12). Since $t$ may be complex,

$$
\delta(t)= \begin{cases}\left|t^{2}-1\right| & \text { on } A_{1} \\ \left|t^{2}+2 t\right| & \text { on } A_{2} \\ |2 t+1| & \text { on } A_{3}\end{cases}
$$


where $A_{1}$ is the union of the region enclosed by $C_{1} \cup C_{3}$ with the region enclosed by $C_{2} \cup C_{4}, A_{2}$ is the reflection of $A_{1}$ in the line $\operatorname{Re} t=-\frac{1}{2}$, and $A_{3}=\mathrm{C}-$ $\left(A_{1} \cup A_{2}\right)$, where

$$
\begin{aligned}
& C_{1}=\left\{-\frac{1}{2}+\frac{\sqrt{3}}{2} e^{i \theta}, 0 \leqslant|\theta|<\frac{\pi}{2}\right\}, \\
& C_{2}=\left\{-\frac{1}{2}+\frac{\sqrt{3}}{2} e^{i \theta}, \frac{\pi}{2}<|\theta|<\pi\right\}, \\
& C_{3}=\left\{1+\sqrt{3} e^{i \theta}, 0<|\theta|<\frac{5}{6} \pi\right\}, \\
& C_{4}=\left\{1+\sqrt{3} e^{i \theta}, \frac{5}{6} \pi<|\theta|<\pi\right\} .
\end{aligned}
$$

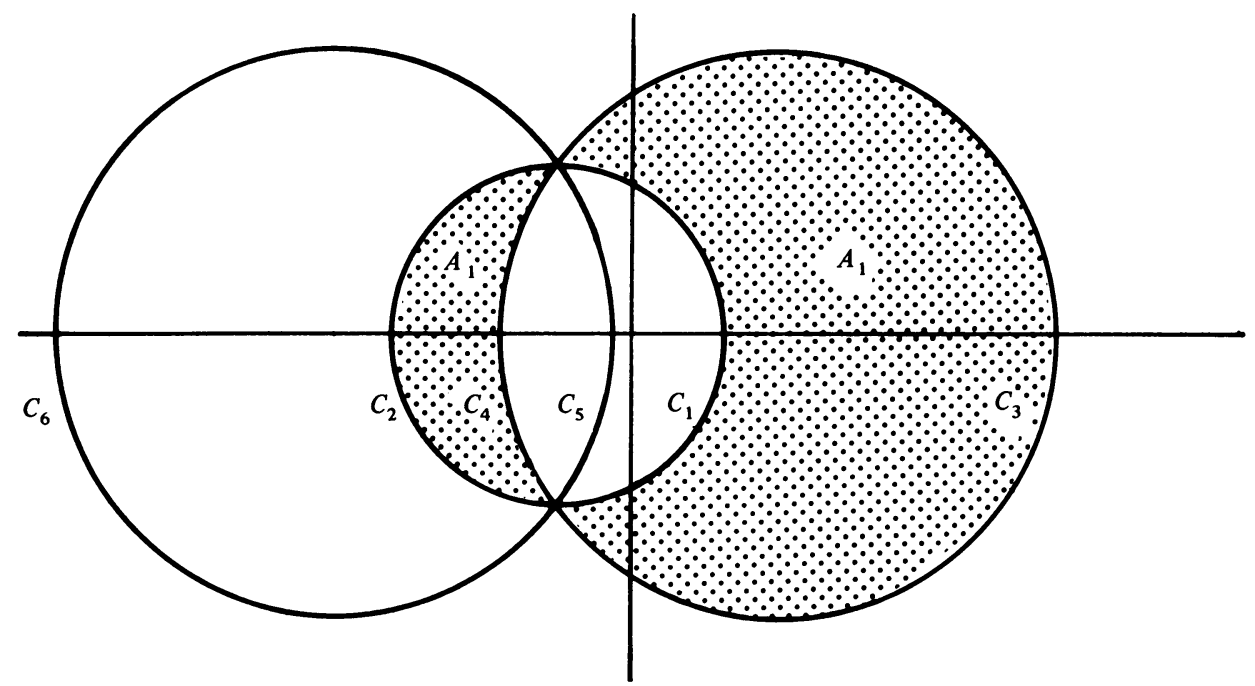

Figure 1

The shaded area in Figure 1 is $A_{1}$. Substitute (13) into (12), use the maximum modulus theorem on $(G(t))^{-1}$ to see that the minimum of $G$ must occur on one of the three circles of Figure 1, and use the method of elementary calculus on each circle, treating $G$ as the appropriate function of $\theta$ on each $C_{i}$. The result is

$$
\min _{t \in \mathbf{C}}|G(t)|=\frac{1}{9} \sqrt{3}
$$

and that the minimum occurs at $t=\omega$ and $\omega^{2}$. Again, the corresponding difference quotients are equivalent; in fact, both are equivalent to difference quotient $d_{\mathbf{2}}$ (see (3)). Notice that, for any nonzero complex constant $c=\rho e^{i \varphi}$, the mapping $(\mathbf{a}, \mathbf{b}) \rightarrow$ $\left(c^{-1} \mathbf{a}, c \mathbf{b}\right)$, gives an equivalent best complex three-point rule minimizing truncation error. Thus the "obvious" best three-point rule here is any one obtained by letting b be the three vertices of any equilateral triangle centered at $x$. (The word "obvious" is in quotes since the corresponding symmetric choice was not the best one in the real case above.)

\section{Roundoff Error.}

Motivation for Theorem 3. In the previous section, evaluations were assumed to be exact. Here we assume that each computation of the function $f$ may be in error 
by as much as $\pm \varepsilon$ where $\varepsilon$ is a small fixed positive quantity. From (5), we see that this generates a roundoff error bounded by

$$
E_{R}=\frac{\left(\left|a_{1}\right|+\left|a_{2}\right|+\left|a_{3}\right|\right) \varepsilon}{h}=\frac{A \varepsilon}{h} .
$$

Fix a difference quotient satisfying (7). From (6) and (7), we see that the dominant term in the truncation error is $c_{4} f^{(4)}(x)(4 !)^{-1} h^{3}$ which is dominated by

$$
E_{T}=\frac{\left|c_{4}\right| f_{4} h^{3}}{4 !}
$$

where $f_{4}=\sup \left\{\left|f^{(4)}(y)\right|: y\right.$ is near $\left.x\right\}$. Define the overall error $E$ by $E=E_{T}+E_{R}$. Elementary calculus shows the $h$ which makes $E$ smallest to be given by

$$
\left\{\begin{array}{l}
h=\left(\frac{8 A \varepsilon}{\left|c_{4}\right| f_{4}}\right)^{1 / 4}, \text { for which } h \text { we have } \\
E=\frac{2^{5 / 4}}{3}\left(A^{3}\left|c_{4}\right|\right)^{1 / 4} f_{4}^{1 / 4} \varepsilon^{3 / 4}
\end{array}\right.
$$

THEOREM 3. Let $d$ be a normalized three-point rule of degree 3. Fix $h$ and restrict a and $\mathbf{b}$ to be real. Then overall error is minimized when $d=d_{1}$. (See Eq. (2).) This minimizing rule is unique up to the transformation $(\mathbf{a}, \mathbf{b}) \rightarrow(-\mathbf{a},-\mathbf{b})$.

Proof. From (15) it follows that we have to minimize $A^{3}\left|c_{4}\right| \cdot$ (Note that (a, b) $\rightarrow$ $\left(c^{-1} \mathbf{a}, c b\right)$ sends $A \rightarrow|c| A,\left|c_{4}\right| \rightarrow|c|^{-3}\left|c_{4}\right|$, so that this quantity is constant over each equivalence class.)

Assume a, b, and $t$ are real. Then

$$
\begin{aligned}
A^{3}\left|c_{4}\right| & =\left(\sum\left|a_{i}\right|\right)^{3}\left|\sum a_{i} b_{i}^{4}\right| \\
& =\left(\frac{\left|t^{3}(t+2)\right|+|2 t+1|+\left|(t+1)^{2}\left(t^{2}-1\right)\right|}{\left|t(2 t+1)(t+2)\left(t^{2}-1\right)\right|}\right)^{3} t^{2}(t+1)^{2} \\
& = \begin{cases}8 t^{8} /\left|(t+1)[(2 t+1)(t-1)]^{3}\right|, & -1<t \leqslant-\frac{1}{2}, 1<t<\infty, \\
8(t+1)^{8} /\left|t[(2 t+1)(t+2)]^{3}\right|, & -\infty<t<-2,-\frac{1}{2}<t<0, \text { and } \\
8 /\left|t(t+1)[(t+2)(t-1)]^{3}\right|, & -2 \leqslant t \leqslant-1,0<t<1 .\end{cases}
\end{aligned}
$$

By elementary calculus we have

$$
\min _{t \in \mathbf{R}} A^{3}\left|c_{4}\right|=\left(\frac{2}{3}\right)^{3} \cdot 2^{4}
$$

and that this minimum occurs at $t=1+\sqrt{3}, 1-\sqrt{3}, \frac{1}{2}(\sqrt{3}-1), \frac{1}{2}(-\sqrt{3}-1)$, $\sqrt{3}-2$, and $-\sqrt{3}-2$. Thus rule $d_{1}$ also minimizes the overall error $E$ among all real normalized three-point rules.

THEOREM 4. Let $d$ be a normalized three-point rule of degree 3. Fix h but allow all variables and functions to be complex-valued. Then overall error is minimized when $d=d_{2}$. (See Eq. (3).) This minimizing rule is unique up to the transformations $(\mathbf{a}, \mathbf{b}) \rightarrow\left(e^{-i \varphi} \mathbf{a}, e^{i \varphi} \mathbf{b}\right), 0<\varphi<2 \pi$. 
Proof. Let $z=2 t+1$. As in the proof of Theorem 3, we must minimize $A^{3}\left|c_{4}\right|=F(z)$ where

$$
F(z)=\frac{1}{16}\left(\frac{|z-1|^{3}|z+3|+16|z|+|z+1|^{3}|z-3|}{\left|z\left(z^{2}-9\right)\right|}\right)^{3} \frac{1}{\left|z^{2}-1\right|}
$$

Inspired by the results of Section 2 (see Theorem 2), we were able to guess that the minimum would occur for $t=\omega$ and $\omega^{2}$, which correspond to $z=\sqrt{3} i$ or $z=-\sqrt{3} i$, where $F=1$. Because of the symmetries $F(z)=F(\bar{z})=F(-z)$, it suffices to show that $F>1$ at every point of the first quadrant, $\mathbf{C}_{+}$, except $\sqrt{3} i$. Let $r=|z|$. For $z \in \mathbf{C}_{+}$and $a \geqslant 0$, we have $|z+a|>r$, so, from (16),

$$
F(z) \geqslant \frac{1}{16}\left(\frac{(r-1)^{3} r+16 r+r^{3}(r-3)}{r\left(r^{2}+9\right)}\right)^{3} \frac{1}{r^{2}+1}=g(r) .
$$

Direct calculation shows $g^{\prime}>0$ on $[9, \infty)$ and $g(9)>1$, reducing the domain of investigation to $C_{+} \cap\{|z| \leqslant 9\}=D$. Finally, a combination of evaluating $F$ on the points of a mesh containing $D$, together with bounding grad $F$ away from the singularities $z=0,1,3$, shows $F>1$ away from $\sqrt{3} i$, while a Taylor expansion of $F$ about $\sqrt{3} i$ shows $F>1$ on the remainder of $D \backslash\{\sqrt{3} i\}$. Thus three-point rule $d_{2}$ also minimizes the overall error $E$ among all normizalized three-point rules.

\section{A Third Rule and Some Remarks.}

Motivation for the Third Rule (see (4)). Estimating the first derivative from an equally spaced table adds the constraint that all three $b_{i}$ be integers. Under these conditions it is clear from formula (11) that the rule given by (4) is best in a number of ways. It is best among integer three-point rules of degree 3 with respect to (i) minimizing $\max \left\{b_{i}\right\}-\min \left\{b_{i}\right\}$, (ii) minimizing $\sum b_{i}^{2}$, (iii) minimizing $\Sigma\left|b_{i}\right|$, et cetera.

History of Three-Point Rules. Essentially all real three-point rules appearing in the literature have been only of degree 2. (However, compare [3, p. 217].) The most prevalent of these is $(-3 f(x)+4 f(x+h)-f(x+2 h)) / 2 h$; see [1]. The complex rule (with $\left.\mathbf{b}=\left(1, \omega, \omega^{2}\right)\right)$ seems to be well known; see [2].

Comparison of Three-Point Rules. In Table 1 below, $f_{4}$ is a bound for the modulus of the fourth derivative near $x$. All derivatives have been normalized to make $\min \left|b_{i}-b_{j}\right|=1$.

Observe that the overall error for the rule $d_{3}$ is only slightly worse than that of the rule $d_{1}$. (By Theorem 3 it must be worse.)

Generalizations. 1. If we use $p>3$ points in our approximating rule, the most we can hope to achieve is

$$
c_{1}=1, \quad c_{0}=c_{2}=\cdots=c_{p}=0 .
$$

This follows from a simple linear algebra argument like the one found in Lemma 2. Such a difference quotient is easily found in the complex case by letting b be the $p$ th roots of unity and solving the Vandermonde system,

$$
c_{1}=1, \quad c_{0}=c_{2}=\cdots=c_{p-1}=0,
$$


for a. Such a quotient appears in [2]. The last equation in (17) holds automatically from the $p$-periodicity. The same result holds in the real case. If $p$ is even, one simply finds the appropriate linear combination of $f(x+h)-f(x-h), f(x+2 h)$ $-f(x-2 h), \ldots, f(x+p h / 2)-f(x-p h / 2)$. This process is essentially Romberg extrapolation. If $p$ is odd, more delicate arguments are required, but Eqs. (17) can still be satisfied.

\section{TABLE 1}

\begin{tabular}{|c|c|c|c|c|c|}
\hline & $\cdots$ & $\begin{array}{l}a \\
\text { b }\end{array}$ & $\ldots \ldots$. & $\begin{array}{l}\text { Truncation Error } \\
\text { (see (14)) }\end{array}$ & $\begin{array}{l}\text { Overall Error } \\
\text { (see (15)) }\end{array}$ \\
\hline $\begin{array}{l}d_{0} \ldots \\
(\operatorname{see}(1))\end{array}$ & $\begin{array}{c}1 \\
1 / 2\end{array}$ & $\cdots$ & $\begin{array}{c}-1 \\
-1 / 2\end{array}$ & $\frac{f_{3} h^{2}}{3 !} \doteq .167 f_{3} h^{2}$ & $\frac{1}{2} 3^{2 / 3} f^{1 / 3} \epsilon^{2 / 3} \doteq 1.04 f_{3}^{1 / 3} \epsilon^{2 / 3}$ \\
\hline $\begin{array}{l} \\
d_{1} \ldots \\
(\operatorname{see}(2))\end{array}$ & $\begin{array}{l}\frac{3-2 \sqrt{3}}{6} \\
\frac{1}{\sqrt{3}}+1\end{array}$ & $\begin{array}{l}\frac{4 \sqrt{3}}{6} \\
\frac{1}{\sqrt{3}}\end{array}$ & $\begin{array}{l}\frac{-3-2 \sqrt{3}}{6} \\
\cdots \cdots \\
\frac{1}{\sqrt{3}}-1\end{array}$ & $\frac{2}{9} \sqrt{3} \frac{f_{4}}{4 !} h^{3} \doteq .016 f_{4} h^{3}$ & $\frac{8}{3^{7 / 4}} f_{4}^{1 / 4} \epsilon^{3 / 4} \doteq 1.17 f_{4}^{1 / 4} \epsilon^{3 / 4}$ \\
\hline $\begin{array}{l}d_{2} \ldots \\
(\operatorname{see}(3))\end{array}$ & $\frac{1}{\sqrt{3}}$ & $\begin{array}{l}\frac{\omega^{2}}{\sqrt{3}} \\
\frac{\omega}{\sqrt{3}}\end{array}$ & $\begin{array}{l}\frac{\omega}{\sqrt{3}} \\
\ldots . . . \\
\frac{\omega^{2}}{\sqrt{3}}\end{array}$ & $\frac{1}{9} \sqrt{3} \frac{f_{4}}{4 !} n^{3} \doteq .008 f_{4} h^{3}$ & $\frac{2^{5 / 4}}{3} f_{4}^{1 / 4} \epsilon^{3 / 4} \doteq .793 f_{4}^{1 / 4} \epsilon^{3 / 4}$ \\
\hline $\begin{array}{l}d_{3} \ldots \\
(\operatorname{see}(4))\end{array}$ & $\begin{array}{l}\frac{32}{40} \\
\frac{3}{3}\end{array}$ & $\begin{array}{l}-\frac{27}{40} \\
\cdots \\
-\frac{2}{3}\end{array}$ & $\begin{array}{l}-\frac{5}{40} \\
\frac{6}{3}\end{array}$ & $\frac{4}{3} \cdot \frac{f_{4}}{4 !} h^{3} \doteq .056 f_{4} h^{3}$ & $\frac{16}{3(375)^{1 / 4}} f_{4}^{1 / 4} \epsilon^{3 / 4} \doteq 1.21 f_{4}^{1 / 4} \epsilon^{3 / 4}$ \\
\hline
\end{tabular}

2. Pass now to the $d$ th derivative, $d \geqslant 2$. To approximate $f^{(d)}(x)$ we now need $p>d+1$. As above, we choose (a, b) in such a way that $d(h)$ is an approximation to the $d$ th derivative, i.e., that $c_{0}=c_{1}=\cdots=c_{d-1}=0, c_{d}=d$ ! (see (6)) and that as many higher order terms be 0 as possible. Again the kind of argument used in Lemma 2 shows that we may hope for $c_{d+1}=c_{d+2}=\cdots=c_{d+p-1}=0$ at most. As in the preceding generalization 1, we may achieve this in the complex case by letting the components of $b$ be the $p p$ th roots of unity. In the real case, however, we cannot always do as well. For example, let $d=2$ and $p=3$. Here

$$
\frac{2}{3}\left[f(x+h)+\omega f(x+\omega h)+\omega^{2} f\left(x+\omega^{2} h\right)\right] / h^{2}=f^{\prime \prime}(x)+\frac{1}{60} f^{(5)}(x) h^{3} \ldots
$$

so $c_{0}=c_{1}=c_{3}=c_{4}=0, c_{2}=2$, as desired, while an easy calculation shows this system to be insoluble with $\mathbf{a}$ and $\mathbf{b}$ real. 
Department of Mathematical Sciences

DePaul University

Chicago, Illinois 60614

1. W. G. BickıEY, "Formulae for numerical differentiation," Math. Gaz., v. 25, 1941, pp. 19-27.

2. J. N. LYNess, "Differentiation formulas for analytic functions," Math. Comp., v. 22, 1968, pp. $352-362$.

3. Herbert E. SAlzer, “Optimal points for numerical differentiation,” Numer. Math., v. 2, 1960, pp. 214-227. 